\title{
Minus 15 Prozent
}

\author{
Eine neue Initiative weist darauf hin, dass nosokomiale Infektionen \\ nach wie vor ein brennendes Thema sind, bei dem es \\ weitere Bemühungen dringend braucht. Christian F. Freisleben
}

Um nicht weniger als 15 Prozent soll die Anzahl nosokomialer Infektionen in Österreich innerhalb der nächsten fünf Jahre gesenkt werden. Das fordert die von der Initiative Sicherheit im OP (SIOP) und der Plattform Patientensicherheit gegründete Initiative minus 15 Prozent. Die heimische Gesundheitspolitik sei hier noch säumig: „Wir fordern ein klares Commitment für dieses Ziel. Zahlreiche internationale Vorbilder zeigen, dass es mit klaren Vorgaben und gemeinsamen Anstrengungen gelungen ist, die gesteckten Ziele der Infektionsverringerung zu erreichen. In Österreich fehlen solche konkreten und verbindlichen Vorgaben“, betont Maria Kletecka-Pulker, Geschäftsführerin der Plattform Patientensicherheit und Direktorin des Ludwig Boltzmann Instituts Digital Health and Patient Safety. Die COVID-Pandemie zeige deutlich, wie viel innerhalb kürzester Zeit zur Infek- tionsprophylaxe möglich ist und auch, wie viele Mittel sowohl auf finanzieller als auch auf struktureller Ebene unmittelbar aktivierbar sind. „Jetzt geht es darum, diesen Schwung zu nützen und bewährte Hygienemaßnahmen unabhängig von COVID beizubehalten und auf breiter Basis zu implementieren“, betont Kletecka-Pulker. Der angestrebte Wert von minus 15 Prozent sei konservativ angesetzt: Das ECDC (European Center of Diseases Control) schätzt, dass sich mit einfachsten Maßnahmen bis zu einem Drittel der nosokomialen Infektionen vermeiden lässt, also durch gute Händehygiene, die Standardisierung von Pflegeprozessen und deren Einhaltung, die Isolation infizierter Patienten und den gezielten Einsatz von Antibiotika - unterstützt durch entsprechend geschulte Spezialkräfte in Medizin, Pflege und Biomedizinischer Analytik. 


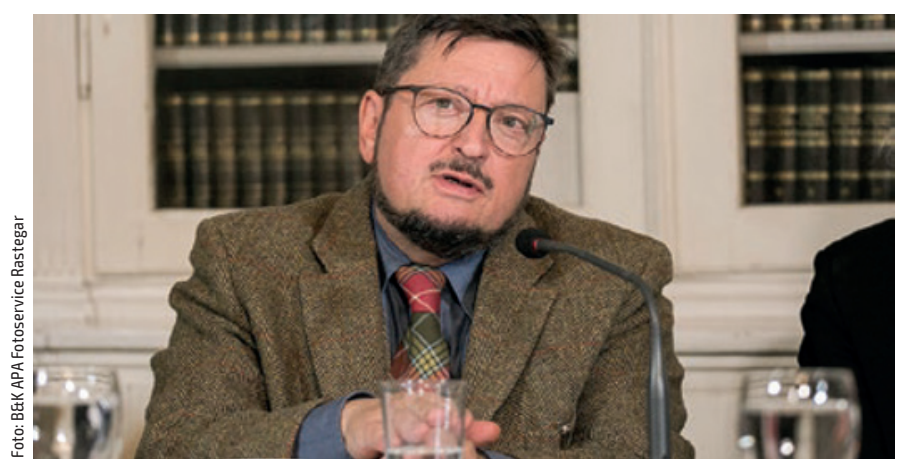

95.000 Menschen erkranken in Österreich im Jahr an nosokomialen Infektionen. Alexander Blacky, Facharzt für Hygiene und Mikrobiologie und Vorstandsmitglied der Österreichischen Gesellschaft für Krankenhaushygiene (ÖGKH), sagt, die zunehmende Verbreitung antibiotikaresistenter Bakterien habe zur Folge, „dass viele dieser Infektionen nicht mehr wirksam behandelt werden können. 4500 bis 5000 Menschen sterben jedes Jahr in Österreich daran“, zehnmal so viele wie im Straßenverkehr.

\section{Problematische Wundinfektionen}

Sehr problematisch seien etwa operationsbezogene Wundinfektionen, die je nach Art der Operation in bis zu zehn Prozent der Fälle auftreten (siehe Kasten). „Gerade in Zeiten, wo der Fokus fast ausschließlich auf einem Virus liegt, ist es wichtig, auf die Bedeutung

\section{COVID UND NOSOKOMIALE INFEKTION}

Berichte aus verschiedenen Krankenhäusern zeigen, dass selbst bei mehrfacher Testung Ansteckungen mit dem Corona-Virus im Spital nicht völlig vermeidbar sind.

Eine aktuelle Analyse ${ }^{1}$ ergibt, dass dafür weniger die Patienten als das Personal verantwortlich ist: Die Infektion verbreitet sich über die Hände der Mediziner und Pflegekräfte und gemeinsam genutzte Geräte wie Fieberthermometer, Blutdruckmess-Manschetten und Stethoskope.

Müssen Patienten mit COVID längere Zeit im Spital betreut werden, steigt gleichzeitig die Wahrscheinlichkeit, dass diese von multiresistenten Keimen betroffen sind, besonders wenn eine Person auf der Intensivstation eine längere Zeit beamtet werden muss. Das Risiko erhöht sich, wenn eine zusätzliche Grunderkrankung vorliegt. Die Keime können etwa über den Katheder in den Körper gelangen, über Infektionsschläuche und einen Tubus. Als Gegenmaßnahme muss dann eine individuelle Antibiotikatherapie zusammengestellt werden

\section{VERMEIDUNG POSTOPERATIVER WUNDINFEKTIONEN}

Aktuelle Studien² zeigen, dass die Wahrscheinlichkeit postoperativer Wundinfektionen in den heißen Sommermonaten besonders hoch ist - um bis zu 30 Prozent höher als im Winter. Dies ist insofern problematisch, da aufgrund hoher Corona-Infektionszahlen zahlreiche Operationen aufgeschoben und eher im Sommer nachgeholt werden.

Als eine der präventiven Maßnahmen wird die Patientendekontamination angeführt: zum einen Schritte, die Patienten selbst etwa durch das Auftragen spezieller Salben umsetzen können, sowie antiseptische Ganzkörperwaschungen und der Einsatz von Nasengel in den Nasenvorhöfen

\section{Hygiene-Facharzt Alexander Blacky: "Unverständlich, dass nichts weitergeht."}

der gesamten Problematik hinzuweisen, die ein Dauerbrenner ist und viel menschliches Leid verursacht sowie sehr hohe Kosten."

Kletecka-Pulker hatte sich zuletzt im Umfeld der Plattform Patientensicherheit auch mit der Haftungsfrage bei nosokomialen Infektionen auseinandergesetzt: „Für den Fall des vermehrten Auftretens von NI in einer Krankenanstalt kann nicht ausgeschlossen werden, dass Versicherungen eine Erhöhung der Versicherungsprämie in ihrer Polizze vorsehen."

Blacky betont, dass es im laufenden Betrieb eines Krankenhauses nötig sei, sich immer wieder dem Themenfeld der Krankenhauskeime zuzuwenden, auch um das Bewusstsein dafür wachzuhalten und entsprechende Maßnahmen abzuleiten. Leitlinien wie die Aktionspläne zur Antibiotikaresistenz (NAP-AMR) und zu multiresistenten Erregern (NAP-MRE) seien zwar sehr wich-

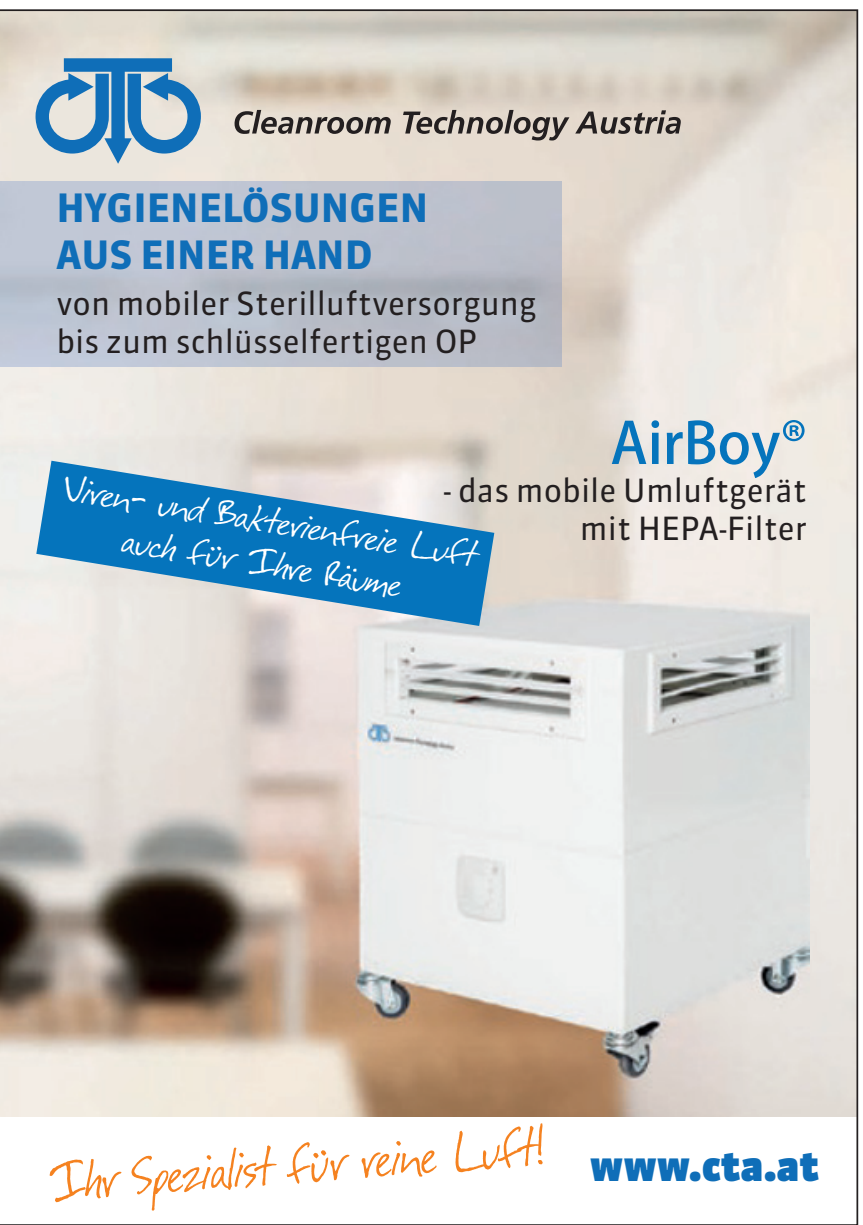


tig und begrüßenswert. Diese würden aber nach wie vor zu wenig Konsequenzen im Spitalsalltag nach sich ziehen. Dies würde auch daran liegen, dass die Umsetzung an einzelnen Spitalsträgern hängt, die dabei sehr unterschiedliche Strategien verfolgen.

Ein Schlüssel zur Umsetzung aktueller Aktionspläne und Richtlinien ist, dass Hygieneteams mit ausreichend Zeit ausgestattet sind und strukturell innerhalb jedes einzelnen Spitals in ihrer Arbeit unterstützt werden. Dies sei trotz mehrfach festgeschriebener Standards und Mindestzahlen bei Weitem nicht in allen Abteilungen der Fall. „Mit zehn Stunden im Monat, die sich aufgrund von Berechnungen in Bezug auf die Bettenzahl ergeben, lässt sich Krankenhaushygiene nur sehr bedingt bis gar nicht vorantreiben. Noch dazu gibt es gerade bei Hygienefachkräften sowohl im ärztlichen als auch im pflegerischen Bereich einen drastischen Personalmangel. "Hier müsste, wie Blacky unterstreicht, mehr investiert werden, was sich dann aber eben in einer Verringerung der Infektionszahlen und damit dann auch in den Kosten rasch niederschlagen würde.

\section{Konzepte in der Schublade}

Endlich konkrete Schritte erhofft sich Blacky zudem dringend auf der Ebene spezialisierter Weiterbildungen: „Wie andere Sonderausbildungen im Pflegebereich hängt leider auch jene zum
Thema Krankenhaushygiene in der Luft, und dies seit Jahren." Die ÖGKH hat dazu längst ein umfassendes Curriculum vorgelegt und mit allen wesentlichen Stakeholdern akkordiert. Dass in den letzten Monaten der Fokus im Gesundheitsministerium

Maria Kletecka-Pulker, Plattform Patientensicherheit: "In Österreich fehlen klare Vorgaben."

woanders lag, sei verständlich - „aber diese gut ausgearbeiteten und durchdachten Konzepte liegen schon deutlich länger in der Schublade, und es ist völlig unverständlich, wieso hier nichts weitergeht“.

\section{Literatur:}

1 Nordling L (2020): Study tells 'remarkable story' about COVID-19's deadly rampage through a South African hospital. Science. Zugang: https://www.sciencemag org/news/2020/05/study-tells-remarkable-story-about-covid-19-s-deadly-rampage-through-south-african. Zugriff: 5.1.2021

2 Eber E (2020): Große Herausforderung auch für die Pflege. ProCare 25, 48-49. Zugang: https://doi. org/10.1007/s00735-020-1211-7. Zugriff: 5.1.2021.

\section{MAG. CHRISTIAN F. FREISLEBEN}

freisleben@gesundheitswirtschaft.at

\section{NORACARE - FÜR HÖCHSTE HYGIENE UND EFFIZIENTE REINIGUNG}

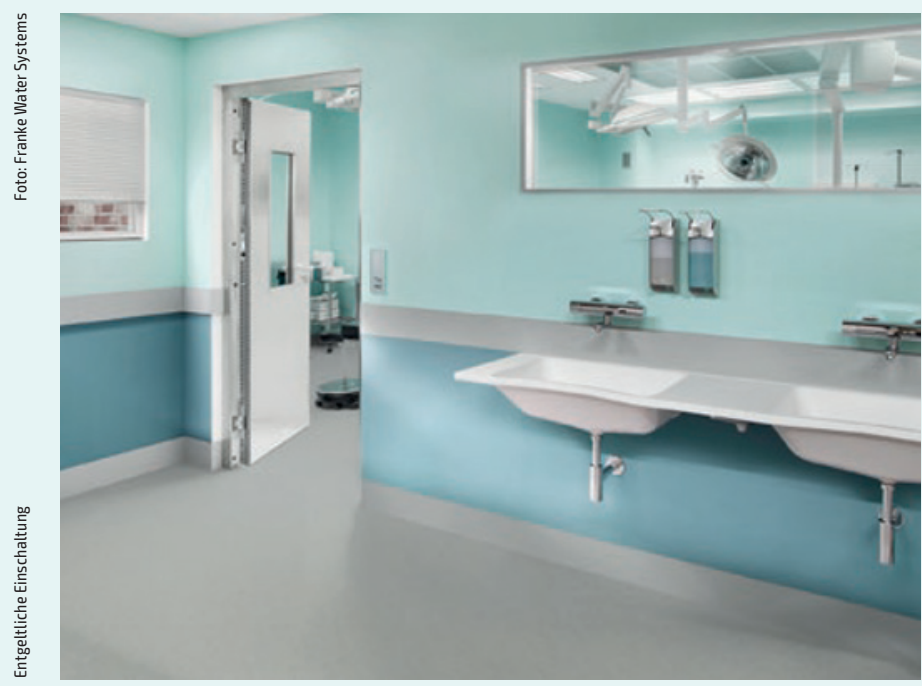

Mit der neuen Produktlinie noracare komplettiert nora flooring systems sein Systemangebot fürs Gesundheitswesen. Der Belag wurde mit seiner innovativen Oberflächentechnologie speziell für Bereiche mit höchsten Hygieneanforderungen und intensivem Einsatz von Desinfektionsmitteln entwickelt und steht für optimale Reinigungsergebnisse. Denn die einfache Reinigung und der kostengünstige Unterhalt von Bodenbelägen sind wichtige Faktoren für einen reibungslosen und wirtschaftlichen Krankenhausbetrieb. noracare ist vor allem für Waschräume und Labore die ideale Lösung, aber auch für Bereiche, in denen die Desinfektionsmaßnahmen aufgrund eines erhöhten Infektionsrisikos gestiegen sind, wie Patienten- und Untersuchungszimmer, Wartebereiche, Flure etc.

Mit noracare entsteht ein Komplettpaket für Kliniken, das sämtliche Einsatzbereiche mit ihren unterschiedlichen Anforderungen an den Boden abdeckt und eine einheitliche Gestaltung im ganzen Haus ermöglicht. Denn das Design von noracare seneo ist auf das der Produktlinie noraplan sentica abgestimmt und überzeugt durch eine seidenmatte Oberfläche. noracare seneo ist mit zwei Millimetern Dicke standardmäßig in 24 Farbtönen verfügbar. Auch in Sachen Umwelt punktet der neue Belag: Er ist bis zu 100 Prozent recycelbar. Wie alle nora Böden ist auch noracare seneo frei von PVC, Phthalat-Weichmachern und Halogenen und leistet einen Beitrag zu einer guten Qualität der Innenraumluft. 